\title{
The Generalized FASS (Full Aperture Seeing Sensor): filling the lower altitudes of the $C_{n}^{2}$ profile
}

\author{
Clémentine Béchet ${ }^{\mathrm{a}}$, Boris Ayancan ${ }^{\mathrm{a}}$, Rodrigo Badinez ${ }^{\mathrm{a}, \mathrm{b}}$, Andrés Guesalaga $^{* \mathrm{a}}$, Marc Sarazin ${ }^{\mathrm{b}}$, \\ Saavidra Perera ${ }^{\mathrm{c}}$, James Osborn ${ }^{\mathrm{c}}$, Richard Wilson ${ }^{\mathrm{c}}$ \\ aPontificia Universidad Católica, Santiago, Chile; 'buropean Southern Observatory, Garching, \\ Germany; ${ }^{c}$ Durham University, Durham, United Kingdom
}

\begin{abstract}
Turbulence monitors based on scintillation are in common use among the astronomical community. One of their weaknesses is that they are blind to turbulences located near the ground where the wavefront traversing these layers have not propagated over sufficiently long distances to generate scintillation patterns with significant contrast. One approach to solve this problem is to allow propagation below the telescope pupil by means of additional optics. The problem with such approach is the diffraction caused by the telescope aperture on the negatively conjugated images. We evaluate the impact of this undesired effect on a recently proposed technique called FASS (Full Aperture Seeing Sensor) that aims to monitor the atmospheric turbulence with a dedicated small telescope of typically 30 -centimeter diameter. The method uses a Fourier analysis of the scintillation patterns to estimate the turbulence strength distribution in altitude. So far, the FASS concept and experimental results have been applied to pupil conjugation only, limiting the reconstruction of the turbulence profile to altitudes higher than $500 \mathrm{~m}$ and requiring a total seeing monitor such as DIMM (Differential Image Motion Monitor) to fill the gap. Our goal is to develop a generalized version of FASS (negative conjugation), which will include the ground layer and where DIMM will not be necessary. In this article, we simulate this configuration, assessing the impact of the diffraction caused by annular aperture affects the profile estimation as a function of the conjugation altitude. We show that one of the features of the Fourier method used for profiling, which is the use of one-dimensional transforms of the image points along concentric rings, makes the technique very robust with respect to the diffraction rings generated by the annular aperture. We also define a method to find the most adequate negative altitude for conjugation, in order to design the future generalized FASS monitor.
\end{abstract}

Keywords: Turbulence profilers, Low noise CCD detectors, Site testing

\section{INTRODUCTION}

Latest generation of low noise CCD detectors offers the possibility of developing atmospheric turbulence monitors based on the processing of high resolution images to replace the well-known MASS and DIMM techniques [1-4]. It is well known that estimating the turbulence at the ground is not simple due to the lack of contrast in the scintillation images generated by layers close to the ground. The combination of MASS and DIMM monitors offers an answer to the problem by taking the information from MASS for the turbulence in altitude and deriving the one at the ground from the global estimation provided by DIMM. In a previous work [5], we have proposed a method to estimate the free atmosphere turbulence, as an alternative to MASS.

We tested the technique against a stereo-SCIDAR [6] monitor developed by Durham University. SCIDAR is a highresolution monitor that also uses scintillation as the principle of probing the atmosphere. During this campaign held at Paranal, (Chile) SCIDAR was installed in one of the $1.8 \mathrm{~m}$ AT telescopes with its optics negatively conjugated. This was not the case for FASS that run with the standard configuration, i. e. conjugated to the pupil (blind to ground turbulence).

Figure 1 shows the estimated profiles for FASS (left) and SCIDAR (right) during the night of April $28^{\text {th }}$, 2016. In the case of SCIDAR, its resolution has been reduced to match that of FASS and for the same reason, its first slab has been deleted. The campaign showed remarkable resemblance between both instruments, despite looking through different air masses.

*aguesala@ing.puc.cl 

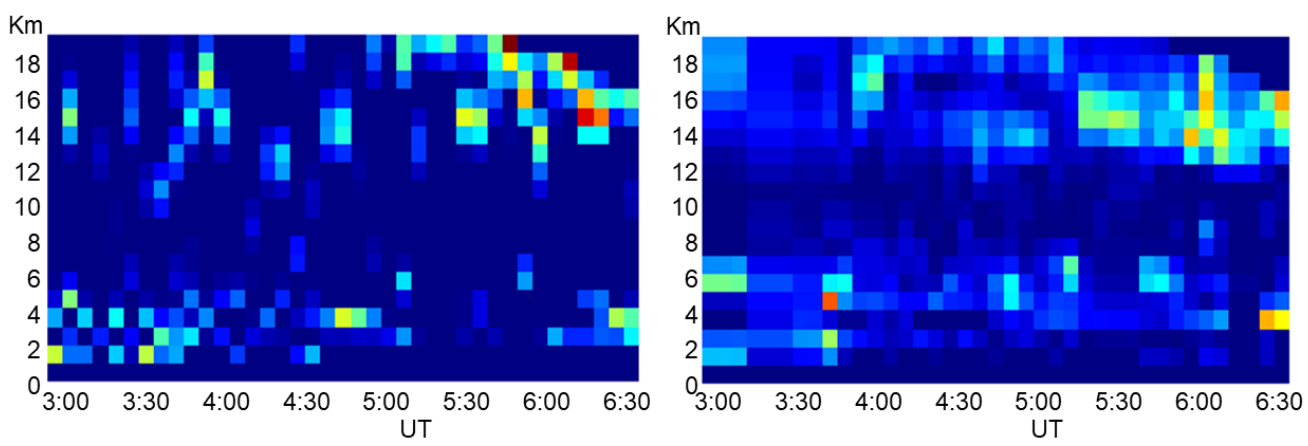

Figure 1. Free atmosphere turbulence profile, first slab not considered. Left: standard FASS; Right: Durham's Stereo SCIDAR. From onsky data, April $28^{\text {th }}, 2016$.

In section 2, we briefly recall the method used for the estimation of the free atmosphere turbulence, as the technical fundamentals for the generalized method remains the same. The reader is referred to [5] for a more detailed description of this technique. Section 3 describe the negative conjugation method used, and finally in section 4 , simulation result that support this design are presented.

\section{THE FREE ATMOSPHERE ESTIMATION}

An obvious approach to extract the speckle size distribution and intensity information from scintillation is to use 2D power spectra from the related images, maximizing the light received by the telescope.


Figure 2. Speckles at the pupil and their 2D power spectra: turbulent layer located at $0.5 \mathrm{~km}$ (top) and $10 \mathrm{~km}$ (bottom).

Figure 2 shows simulated pupil images of scintillation for a $1 \mathrm{~m}$ telescope caused by turbulence located at $500 \mathrm{~m}$ (top) and $10,000 \mathrm{~m}$ (bottom) for a seeing of 0.2 " and their associated two-dimensional power spectral density. The distinctive patterns in the two cases are obvious, with a speckle size that is proportional to the altitude of the layers that according to the Fresnel law is, with $h$ being the altitude of the layer and $\lambda$ the wavelength of the source (here we have monochromatic light at $500 \mathrm{~nm}$ ). So, for the two layers considered: ds @ $0.5 \mathrm{~km}=1.6 \mathrm{~cm}$ and ds @ $10 \mathrm{~km}=7.1 \mathrm{~cm}$. A clear difference exists in the distribution of the power spectra, where smaller speckles populate higher frequencies whereas large speckles caused by high altitude turbulence will tend to concentrate the energy at the centre of the spectrum. This characteristic is used to generate spectral functions that span the range of spatial frequencies generated by the speckles.One of the main requirements for FASS is to apply the technique to small, transportable and low-cost telescopes. This restriction makes the $2 \mathrm{D}$ approach impractical as the larger speckle sizes would be truncated with the subsequent distortion when measuring their spatial frequencies. 
Figure 3 shows pupil images collected from a telescope (Celestron C9.25 XLT, 23.5cm). The images show two different cases of scintillation. The case on the left is populated with speckles of a couple of pixels in size whereas in the second case, two different type of speckles appear; a low spatial frequency pattern (from higher altitude layers) and a high frequency one. In the latter case, the aperture crops the larger speckles, so the information in the radial direction is lost.
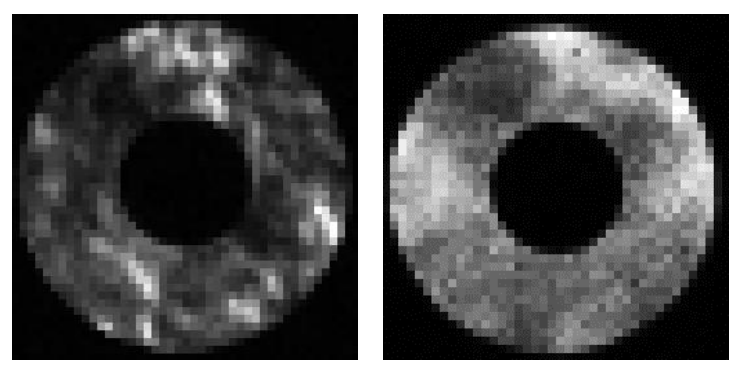

Figure 3. Two different cases of scintillation from on-sky data (September $27^{\text {th }}$ and $29^{\text {th }}, 2015$ ); one dominated by low altitude turbulence (left) and the other with larger speckles corresponding to high altitude turbulence and a high frequency granulation due to noise or a low altitude layer

A solution for this problem that still use a large portion of the incoming light is to reduce the spatial frequency analysis to one dimension only by scanning the annulus in concentric rings. Figure 4 describes this approach, where by resampling the image along concentric rings in the pupil annulus, a longer dynamic range is achieved and larger speckles can perfectly fit. For example, a ring defined by a radius of $r=10 \mathrm{~cm}$, would generate a sampled perimeter of $2 \pi r \approx$ $60 \mathrm{~cm}$, which can accommodate up to three complete cycles of $10 \mathrm{~cm}$ speckles (resulting after propagating a wavefront from a layer at $18 \mathrm{Km}$ ). The Cartesian grid formed by the detector pixels is resampled via interpolation, using a polar grid that unravels one or more concentric rings. In Fig. 4, five rings are unraveled to form a matrix of 128x5 pixels. The lines of this matrix (length 128) are Fourier transformed and later averaged.

By calculating the power spectra of each linear vector in the unravelled array and repeating this procedure for a large number of images, a spectrum is obtained for the subsequent profile estimation. This spectrum is matched to a linear combination of reference spectra simulated by propagation light from single layers at different altitudes.

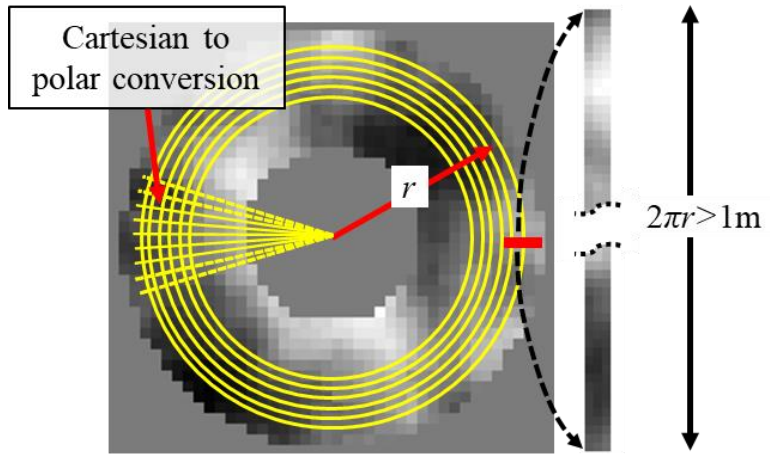

Figure 4. Pupil images for a 9.5" telescope. Transformation from a Cartesian to a polar grid repeated for five concentric rings, with 128 angular divisions (left) and unraveling the rings into linear arrays for a later circular 1-D Fourier transform and average (right).

The benefits of unravelling the annulus is three-fold:

- It reduces the impact of diffraction rings from inner and outer pupil edges, especially at large negative conjugation altitudes. This problem is the core of this article and will be treated in detail in the next sections

- Larger speckles generated by higher altitude layers can be detected due to sampling along the $2 \pi r$ rings ( $r$ is the central radius of the resampled rings)

- The Fourier-transform is performed for a circular, continuous 1-D vector along the concentric rings shown in Fig. 4. This avoids discontinuities in the Fourier-transformed vector that otherwise, would had caused diffraction and leakage over the whole spectrum. 


\section{THE GENERALIZED (NEGATIVE CONJUGATION) SOLUTION}

Our long-term objective is to develop a generalized version of FASS where scintillation is imaged at negative conjugation, allowing the extra propagation to develop speckles with a significant contrast to be detected by the later processing. Achieving this aim would make accompanying monitors such as DIMM unnecessary.

The generalized FASS version we proposed, is depicted by the optical schematics shown in Fig. 5, where the light from the telescope aperture is transferred to the detector, generating an image of the scintillation at negative conjugation. At the CCD plane, this system forms a pupil image from a plane located the required distance $\left(Z_{\text {conj }}\right)$ behind the telescope mirror $\left(l_{1}\right)$. The telescope mirror $\left(L_{1}\right)$ forms a virtual image of the plane of focus $\left(P_{1}\right)$ at a distance $l_{1}$ ' behind the telescope mirror. The field lens $L_{2}$ re-images this virtual object to form the pupil image at the CCD plane. For the generalized FASS the field lens $L_{2}$ is located at $l_{2}$ ' from the CCD plane. A virtual image of a plane at $1.2 \mathrm{~km}$ (selected negative conjugation for FASS) is formed $l_{2}$ in front of $L_{2}$ and this virtual object is imaged by $L_{2}$ onto the CCD detector.

The optics also comprises a bandpass optical filter, $160 \mathrm{~nm}$ width and centered at the star's spectral maximum.

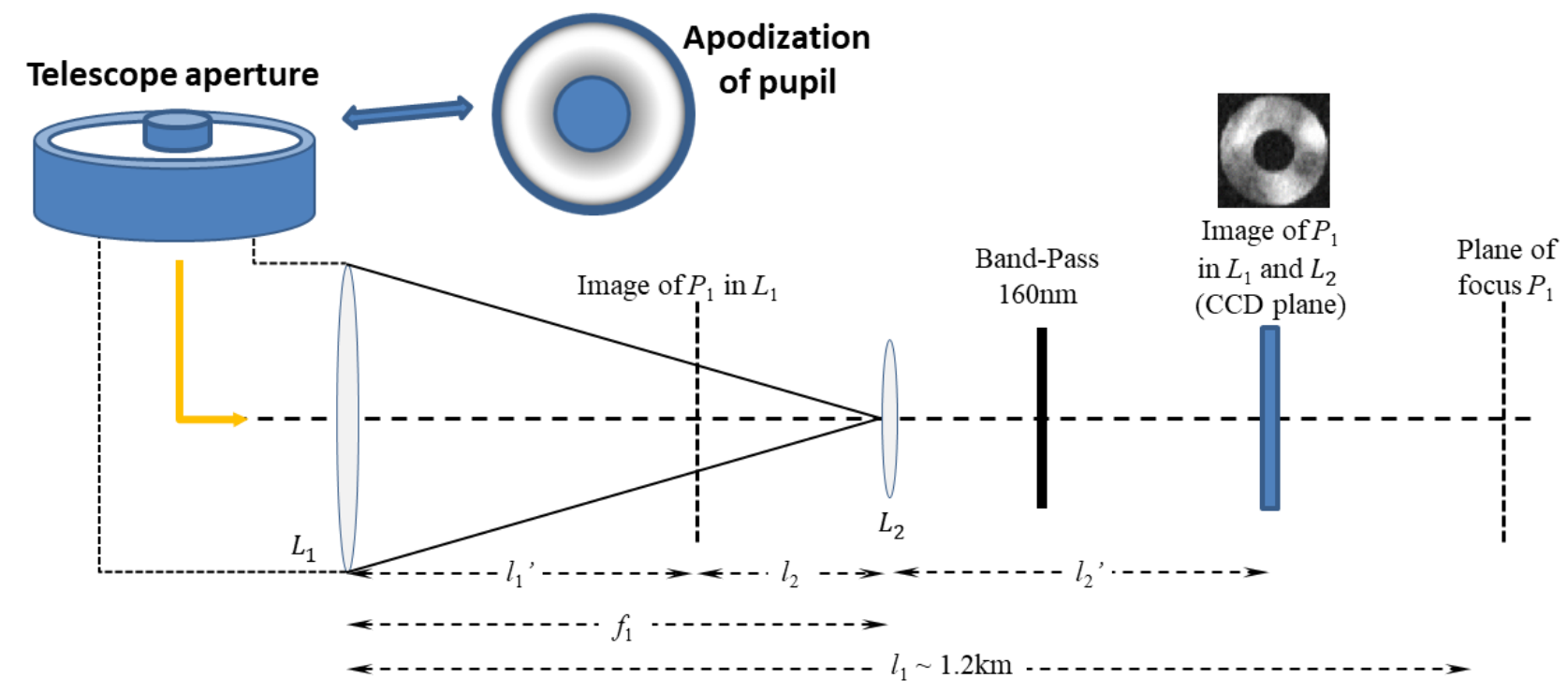

Figure 5. Generalized FASS configuration.

\subsection{Apodization of the pupil}

One of the disadvantages of negative conjugation is the diffraction caused by the annulus edges. In simulations, we found that is highly convenient to apodize the pupil screen, as it can substantially reduce the diffraction caused by these aperture discontinuities.

Two types of apodization filter where tested; one based on the multiplication of two super-Gaussian functions and the other, a Tukey window [7].

i) Super-Gaussian: $W(r)=\left(1-\exp \left(-\left|\frac{y}{D}\right|^{n_{\text {iñ } \mathrm{f}}}\right)\right) \cdot \exp \left(-\left|\frac{y}{D}\right|^{n_{\mathbb{E x t}}}\right)$

ii) Tukey window: $W(r)=W_{1}(r) \cdot W_{2}(r)$,

where $\quad W_{m}(r)=\left\{\begin{array}{cc}\frac{1}{2}\left[1+\cos \left(\frac{2 \pi}{D_{n n}}\left[r-\frac{D_{n n}}{2}\right]\right)\right] & 0 \leq r<\frac{D_{n n}}{2} \\ 1 & \frac{D_{m n}}{2} \leq r<1-\frac{D_{m n}}{2} \\ \frac{1}{2}\left[1+\cos \left(\frac{2 \pi}{D_{m n}}\left[r-1+\frac{D_{m n}}{2}\right]\right)\right] & 1-\frac{D_{m n}}{2} \leq r \leq 1\end{array}\right.$

The subindex $m=\{1,2\}$ stands for each of the two phasescreens used for apodizing the inner and outer edges respectively. 
Figure 6 shows the effect of apodizing the telescope aperture. The top row contains the simulated aperture mask for a $30 \mathrm{~cm}$ telescope (extreme left) and then, the image generated at four different altitudes (negative conjugation) where strong diffraction is observed in all of them. As mentioned above, this is the disadvantage of negative conjugation and although our approach of sampling concentric rings (see Fig. 4) reduces the impact on the method accuracy, it still represents a major source of disturbance.

The bottom row in Fig. 6 shows the apodization mask printed on the pupil (extreme left) and the resulting images for the same altitudes as the top row. Despite the loss in the effective area available for collecting light, a significant reduction in the strength of the diffraction rings is observed.



Figure 6. The diffraction problem. Top row: telescope aperture and diffraction patterns for four different altitudes. Bottom row: apodization mask and corresponding diffraction patterns.

We have found that $Z_{\text {conj }}=-1.2 \mathrm{~km}$ is a good compromise between speckle contrast, diffraction and dynamic range of observable altitudes. Figure 7 shows a cut along the intensity annulus with and without apodization shows a substantial reduction in the diffraction rings. The rings to be sampled (Fig. 4) are to be located within radius pixels 20 to 33.

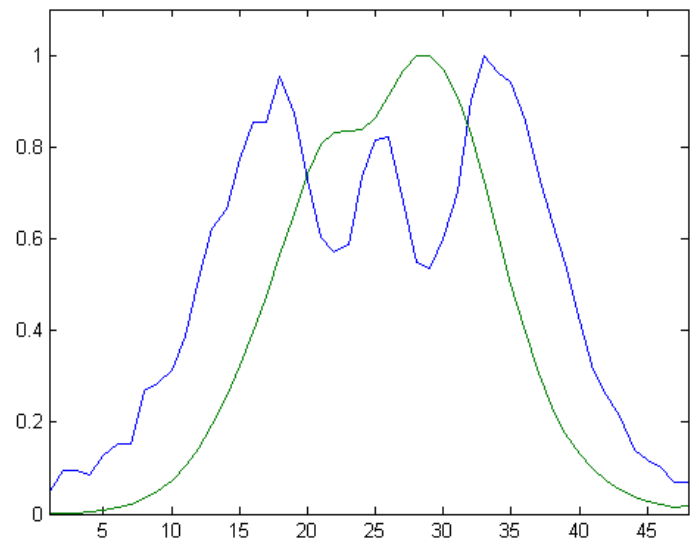

Figure 7. Cut along annulus for $Z_{\mathrm{conj}}=-1.2 \mathrm{~km}$ for the cases with and without apodization mask

The effectiveness of the apodization is presented in Fig. 8 where a super-Gaussian mask is used for a negative conjugation of $Z_{\text {conj }}=-1.2 \mathrm{~km}$. The left image shows the raw intensity obtained, where a diffraction ring can still be observed. The central image corresponds to the average of the intensity images integrated along a sequence of 30,000 frames. By dividing pixel-to-pixel the former by the latter and subtracting the average value, a normalized image is obtained (right image). The diffraction ring has almost completely vanished inside the annulus delimited by the circumference lines. 


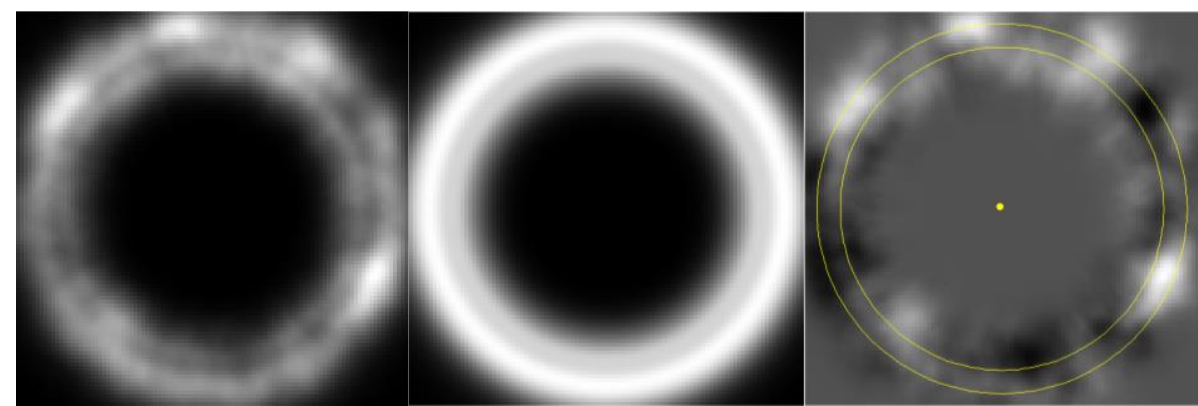

Figure 8. Normalization of intensity images. Left: raw image at conjugation distance. Centre: average of 30,000 frames: Right: normalized image

\section{SIMULATION RESULTS}

Once the full set of images are processed (30,000 in our case) to obtain a spectral average, a fitting with the reference (theoretical) spectra follows. Figure 9 shows the theoretical spectra for the twelve reference layers used in this profile reconstruction process. See [5] for a detailed explanation.

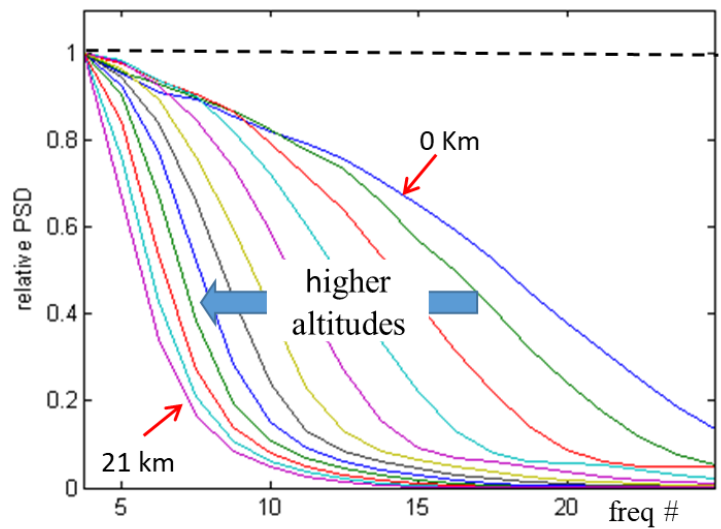

Figure 9. Total of 12 weighting functions from the averaged spectra. Only 18 spectral points suffice for a profile estimation

The profile is calculated by fitting the measured spectrum against a linear combination of the theoretical spectra (Fig. 9) pre-computed for 12 altitude, namely: $Z_{\text {ref }}=\{0,4.0,1.1,2.1,3.4,5.0,6.9,9.1,11.6,14.4,17.5,20.9\} \mathrm{km}$.

Figure 10 shows the response functions for each of the 12 slabs computed from light propagated through individual layers at different altitudes. A clear improvement is observed when compared to MASS responses shown on the right panel, not only in terms of number of slabs (6 against 12 for FASS), but also in the estimation errors.

Finally, Fig. 11 shows the result for the estimation of an arbitrary profile, with five layers chosen at random altitudes. FASS detects seven slabs that describe the real profile. The two additional estimated slabs appear to represent real layers located at altitudes that not coincide with the pre-defined FASS slabs. 



Figure 10. Left: FASS response for 12 slabs. Right: Classical MASS response for six slabs (Kornilov et al, 2003). The FASS method shows a substantial improvement in the estimation error, together with twice the number of altitude slabs and a reliable value for the ground turbulence.

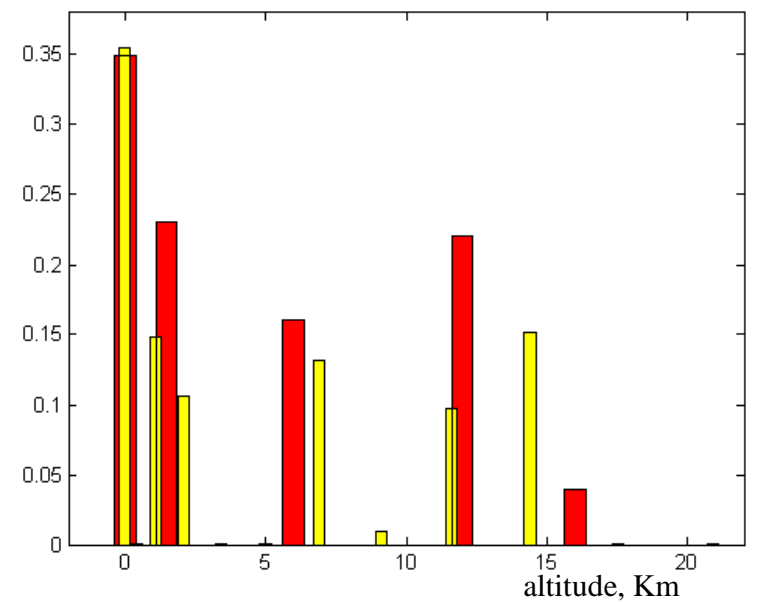

Figure 11. Estimation of an arbitrary profile with layers at random altitudes. The wider bars represent the real fractional $r_{0}$.

\section{CONCLUSIONS}

We have demonstrated that negative conjugation is possible for small telescopes, and that the negative impact of the aperture diffraction can be effectively handled by apodization and a normalization of the intensity image. An adequate conjugation altitude was found at $Z_{\text {conj }}=-1.2 \mathrm{~km}$, representing a good compromise among speckle contrast, noise, pixel size, diffraction and dynamic range of observable altitudes.

Although it was not addressed in this article, the effect of tip and tilt (and vibrations) is not negligible in the accuracy of this generalized approach, but can be corrected via a correct choice of the rings to be processed and a shifting to center the scintillation images

We expect to initiate the construction of the instrument in the near future.

\section{REFERENCES}

[1] Kornilov V.,Tokovinin A.,Voziakova O.,Zaitsev A.,Shatsky, N.,Potanin S., Sarazin M., 2003, Proc. SPIE, 4839,837

[2] Kornilov V., Tokovinin A., Shatsky, N.,Voziakova O., Potanin S., Safonov B., MNRAS, 382, 2007

[3] Tokovinin A., 2003, J. Opt. Soc. Am. A., 20, 686

[4] Tokovinin A., Kornilov V., 2007, MNRAS, 381, 1179 
[5] Guesalaga A., Perera S., Osborn J., Sarazin M., Neichel B., Wilson R., 2016, Proc. SPIE 9909, 99090H

[6] Shepherd H.W., Osborn J., Wilson R.W., Butterley T., Avila R., Dhillon V.S. and Morris T.J., MNRAS, 2014

[7] Bloomfield P., Fourier analysis of time series: an introduction. New York: Wiley-Interscience, 2000

\section{ACKNOWLEDGEMENTS}

This project has been supported by grant Fondecyt-1160236 and grant Anillo-1417 